\title{
Cement Plug Fragmentation Following Percutaneous Cementoplasty of the Bony Pelvis: Is it a Frequent Finding in Clinical Practice?
}

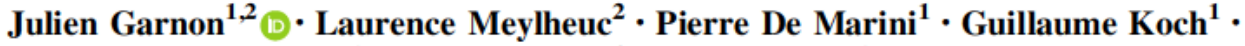 \\ Roberto Luigi Cazzato $^{1} \cdot$ Bernard Bayle $^{2} \cdot$ Afshin Gangi $^{1}$
}

\begin{abstract}
Purpose To report the rate of fragmentation of the cement plug following percutaneous cementoplasty with polymethylmethacrylate (PMMA) in the bony pelvis (i.e., pelvic bones or sacrum).

Materials and Methods Post-interventional and follow-up CT scans of 56 patients ( 36 men; mean age of $68.4 \pm 15.4$ ) with a total of 98 percutaneous cementoplasty procedures were analyzed. Indications for treatment included painful malignant tumors $(42.9 \%$; $42 / 98)$ and insufficiency fractures $(57,1 \%$; 56/98). Fragmentation of PMMA was recorded for each cement plug.

Results Mean interval between the procedure and the last available CT scan was $29.3 \pm 18.8$ months. There was no
\end{abstract}

Julien Garnon

juliengarnon@gmail.com

Laurence Meylheuc

laurence.meylheuc@insa strasbourg.fr

Pierre De Marini

pierre.demarini@gmail.com

Guillaume Koch

guillaume.koch@gmail.com

Roberto Luigi Cazzato

gigicazzato@hotmail.it

Bernard Bayle

bernard.bayle@unistra.fr

Afshin Gangi

afshin.gangi@chru strasbourg.fr

1 Department of Interventional Radiology, Nouvel Hôpital Civil, 1, place de l'hôpital, 67096 Strasbourg Cedex, France

2 UMR 7357, CNRS, ICube, INSA Strasbourg, Université de Strasbourg, 67091 Strasbourg, France significant difference between the length of follow-up of malignant lesions (27.6 \pm 15.1 months) and insufficiency fractures $(29 \pm 20.5$ months $)(p=0.69)$. Fragmentation was diagnosed following $2 / 98(2 \%)$ procedures, both in the malignant lesions group. The time intervals between the procedure and the first visualization of cement fragmentation were 6 for the first and 24 months for the second patient.

Conclusion Fragmentation of the PMMA plug following percutaneous cementoplasty in the bony pelvis is a rare finding at midterm follow-up. It was only observed in cementoplasty performed in malignant lesions and seems to be more a consequence of local mechanical stresses than as a result of porosity.

Keywords Cementoplasty · PMMA · Fragmentation · Breakage $\cdot$ Bony pelvis

\section{Introduction}

Percutaneous cementoplasty with polymethylmetacrylate (PMMA) bone cement is an effective treatment for painful spinal or extraspinal bone metastases and spinal osteoporotic compression fractures [1 3 ]. Because of the mechanical properties of PMMA, cementoplasty also has the ability to achieve consolidation of fractures and impending fractures in locations predominantly withstanding compression stresses [4 6]. In the bony pelvis notably, cementoplasty has been indicated to stabilize insufficiency/pathological fractures of the sacral ala and impending fractures of the acetabulum $[2,5,7,8]$. Once the cement has been deposited and has cured inside the bone, 
the plug of PMMA is not expected to undergo remodeling nor resorption over time [9 11 ]. However, in vivo changes to the morphology of the cement volume may occur. Various potential causes of such findings have been reported in the experimental and clinical literature: infection, mechanical breakage, cracking and foreign body reaction (FBR) [12 18]. Most of the data on the causes and consequences of cement fragmentation come from the orthopedic literature, as loosening and failure of arthroplasties are relevant clinical issues [17, 18]. There are only sporadic case reports of PMMA breakages in the spine following vertebroplasty [12, 19, 20]. Although it does not seem to be a frequent clinical problem, literature on cement fragmentation after percutaneous cementoplasty in the bony pelvis is still lacking. The purpose of the present study is therefore to report the rate of PMMA fragmentation following percutaneous cementoplasty in the bony pelvis (i.e., pelvic bones or sacrum) and discuss the underlying mechanisms and consequences.

\section{Materials and Methods}

This is a single center retrospective observational study. Institutional review board approval was obtained for the retrospective evaluation of the radiological data. All patients gave informed consent to undergo the intervention.

\section{Patient Selection}

Retrospective chart review of all cementoplasty procedures performed with PMMA in the sacrum or the pelvic bones between January 2010 and October 2019 for the management of benign insufficiency fractures or malignant tumors was conducted. Patients who benefited from the combination of screw fixation and cementoplasty during the same intervention were excluded. In order to compare the radiological findings with the same modality, only the patients who were treated under combined CT-fluoroscopic guidance (thus with an immediate post procedural CT-acquisition), and who were restaged (whatever the indication) with a thoraco-abdominopelvic, abdominopelvic or pelvic CT scan during follow-up were included in the present study.

\section{Procedures}

All interventions were performed under combined CT and fluoroscopic guidance using a mobile C-arm prior to 2017 (Somatom Definition AS and Arcadis Orbit, Siemens, Erlangen, Germany) and an angio-CT after 2017 (Alphenix 4DCT, Canon Medical, Japan). Cementoplasty was performed under conscious sedation, regional spinal anesthesia or general anesthesia, in the prone or supine position depending on the location and number of cement injection(s). PMMA was manually mixed (Osteopal V, Heraus medical) according to manufacturer's recommendations and injected through a 10 or $15 \mathrm{~cm} 10 \mathrm{G}$ beveled vertebroplasty needle (Vertebroplasty special needle set, Optimed, Germany) using a dedicated injection device (Cemento MP, Optimed, Germany). Injection was performed under continuous fluoroscopic monitoring and intermittent CT-acquisitions. An immediate post-interventional volumetric CT scan was systematically acquired to ensure optimal cement distribution and rule out any immediate complication.

\section{Data Collection and Analysis}

Pre- and last available postoperative CT scans were reviewed side-by-side on a dedicated workstation (Osirix, Pixmeo, Switzerland) by two interventional radiologists with 4 and 10 years of experience in percutaneous cementoplasty $(\mathrm{XX}, \mathrm{XX})$ on $1 \mathrm{~mm}$ thickness axial, coronal, sagittal images and $15 \mathrm{~mm}$ maximum intensity projection (MIP) axial, coronal and sagittal images, with a $3000 \mathrm{HU}$ window level 10,000 HU window width setting (Fig. 1). Each radiologist reviewed the images independently. Differences were resolved by consensus. The following features of the PMMA plug and surroundings were collected: (1) apparition of a fracture line inside the cement plug on $1 \mathrm{~mm}$ thickness images; (2) modification of the shape of PMMA on MIP images; (3) identification of PMMA distant to the original cement volume on MIP images; (4) stability, consolidation (defined as the restoration of a normal cortical structure), worsening or apparition of a bone fracture in the area of cementoplasty on $1 \mathrm{~mm}$ thickness images; (5) stability, regression, increase or apparition of osteolysis around the cement on $1 \mathrm{~mm}$ thickness images (Fig. 2). According to descriptions in the literature, fragmentation of PMMA was recorded in case of an apparition of a fracture line inside the cement plug and/or if the shape of PMMA was modified and/or if PMMA was identified distant to the original volume $[11,19,20]$. Should fragmentation be present, the filling rate of the osteolysis was calculated using volumetric evaluation, and the entire patient's medical history was reviewed in order to look for signs of local infection and assess the pain level using the visual analog score before the intervention and at the last clinical evaluation [21].

\section{Statistics}

Descriptive statistics were used to present results. Categorical variables were expressed as absolute numbers and percentages. Continuous variables were expressed as 


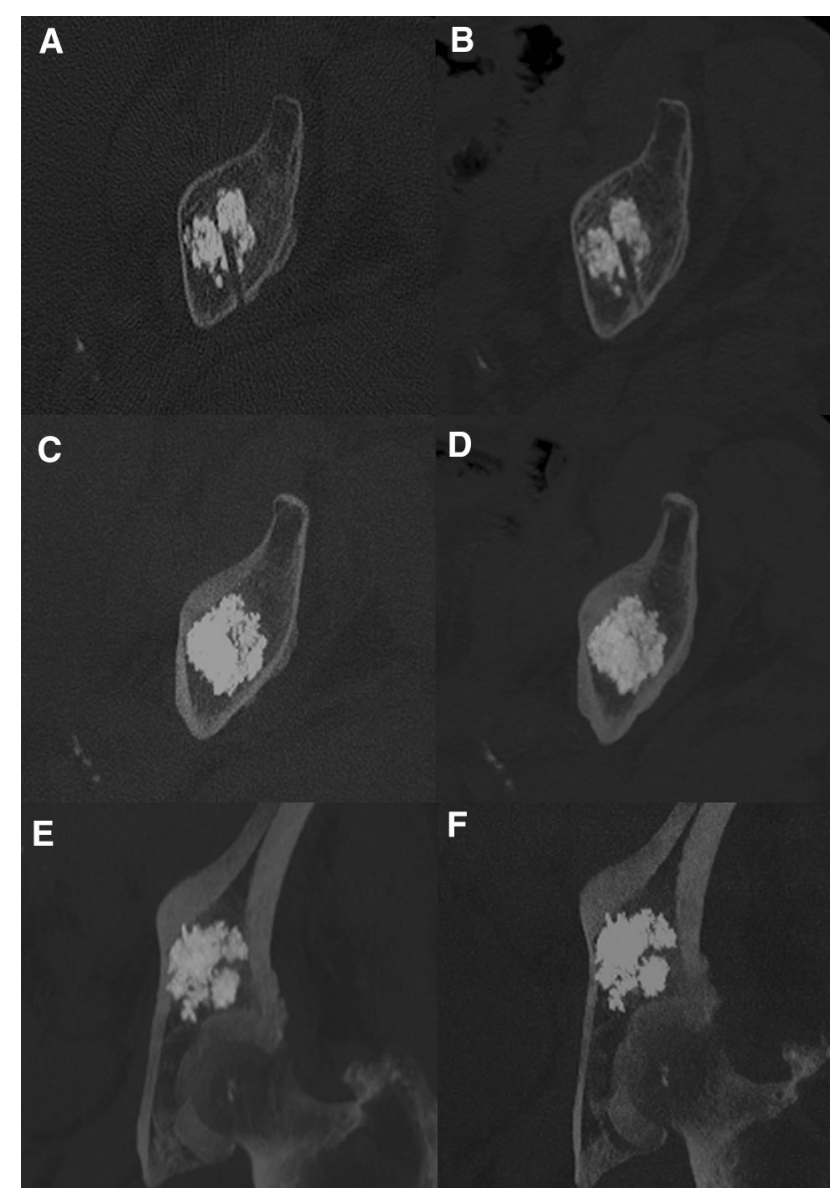

Fig.1 Side by side comparison. Immediate post intervention axial $1 \mathrm{~mm}$ thickness CT $\operatorname{scan}(\mathbf{A})$ and 23 months follow up $1 \mathrm{~mm}$ thickness CT scan (B). Immediate post intervention axial (C) and coronal (D) $15 \mathrm{~mm}$ MIP CT scan and 23 months follow up axial (E) and coronal (F) $15 \mathrm{~mm}$ MIP CT scan CT. Analysis was also performed in $1 \mathrm{~mm}$ thickness coronal and sagittal and $15 \mathrm{~mm}$ MIP sagittal images (not shown)

means with standard deviations and ranges. Comparison of the length of follow-up and the rate of cement fragmentation between malignant lesions and benign insufficiency fractures was made using a t-test. A $p$ value $<0.05$ was considered statistically significant. Statistical analysis was performed by using R v3.6.3 (R Foundation for Statistical Computing, Vienna, Austria-copyright mark).

\section{Results}

One-hundred and eighty-nine patients were treated with percutaneous cementoplasty during the study period. After exclusion of patients treated with Cone-beam CT guidance and patients either lost to follow-up or without a restaging CT scan, the final study population consisted of 56 patients (36 men, 20 women) with a mean age of $68.4 \pm 15.4$ (range 29 92), in which a total of 98 percutaneous target lesions/tumors were treated in 56 sessions $(1.75 \pm 0.8$ cementoplasty procedures per intervention; range 14 ). Indications for treatment were painful malignant tumors $(42.9 \% ; 42 / 98)$ and insufficiency fractures $(57,1 \% ; 56 / 98)$. Radiation therapy was performed prior to the cementoplasty procedure in 19/42 (45.2\%) and scheduled after the intervention in 11/42 (26.2\%) malignant lesions. There was no systematic planification of radiation treatment for the remaining 12/42 (28.6\%) lesions. Most frequent locations of cement injection were the sacral ala $(60.2 \% ; 59 / 98)$ and the acetabulum $(21.4 \% ; 21 / 98)$. Other cemented regions $(18.4 \% ; 18 / 98)$ included the iliac wing $(n=13)$, the body of S1 $(n=3)$ and the ischium $(n=2)$. Cementoplasty of the sacral ala was predominantly performed for insufficiency fractures $(83 \% ; 49 / 59)$, while acetabuloplasty was mostly applied for malignant cases $(91 \% ; 19 / 21)$. A cortical fracture was noted in 39/56 (69.6\%) of the insufficiency fractures $(69.6 \%)$. For malignant cases, lesions were predominantly osteolytic (95\%; 40/42). Cortical destruction and pathological fractures were present in 19/42 (45.2\%) and 1/42 (2.4\%) cases, respectively. Percutaneous radiofrequency ablation was performed during the same procedure in 1/42 (2.4\%) malignant cases. A flowchart of patient selection and lesions is presented in Fig. 3.

Mean interval between the procedure and the last available CT scan was $29.3 \pm 18.8$ months (range 677 ). There was no significant difference in the length of followup of malignant lesions (27.6 \pm 15.1 months) vs insufficiency fractures $(29 \pm 20.5$ months $)(p=0.69)$.

Analysis between the 2 readers was $100 \%$ concordant regarded the 5 collected items. A fracture with a modified PMMA shape was demonstrated in 2/98 (2\%) cementoplasty procedures, both in malignant peri-acetabular lesions. PMMA distant to the initial cement volume (in the hip joint) was identified in one case. Hence, fragmentation was diagnosed in $2 / 98(2 \%)$ cementoplasty procedures, all in the malignant lesions group ( $2 / 425 \%)$. The filling rate of the osteolysis by the cement was $36 \%$ and $49 \%$. None of these two cases benefited from concomitant thermal ablation. Both lesions received additional external radiation therapy within the month following PMMA injection. The time intervals between the procedure and the first visualization of cement fragmentation were 6 and 24 months, respectively. In one case, the last restaging CT scan showed collapse of the hip joint and regression of the osteolysis around the cement (most likely secondary to systemic treatment and radiation therapy), while the other case was associated with an increase in osteolysis and apparition of a pathological fracture due tumor progression (Figs. 4, 5). There was no sign of infection in any of these 2 cases. Pre-intervention pain level scores were $9 / 10$ and 6/10; corresponding pain scores at last available follow-up were $7 / 10$ and $8 / 10$, respectively. 
Fig.2 Patterns defining cement fragmentation in our study. A Identification of a fracture line inside the cement plug. B Modification of the shape of the cement volume.

C Identification of PMMA distant from the original cement plug
A
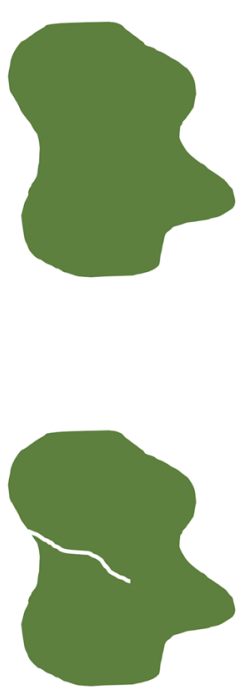

B
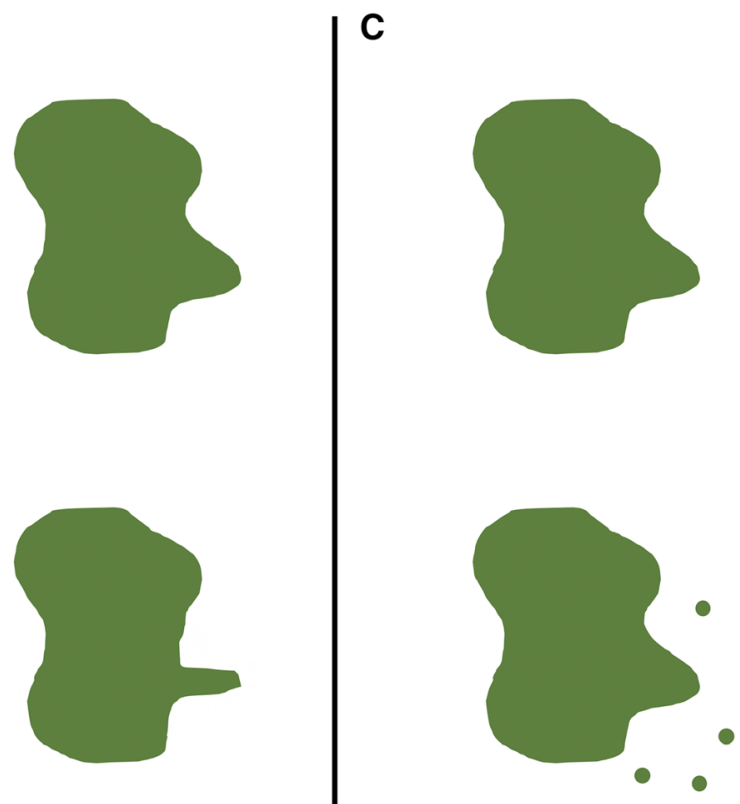

Fig.3 Flowchart of patient selection and lesions

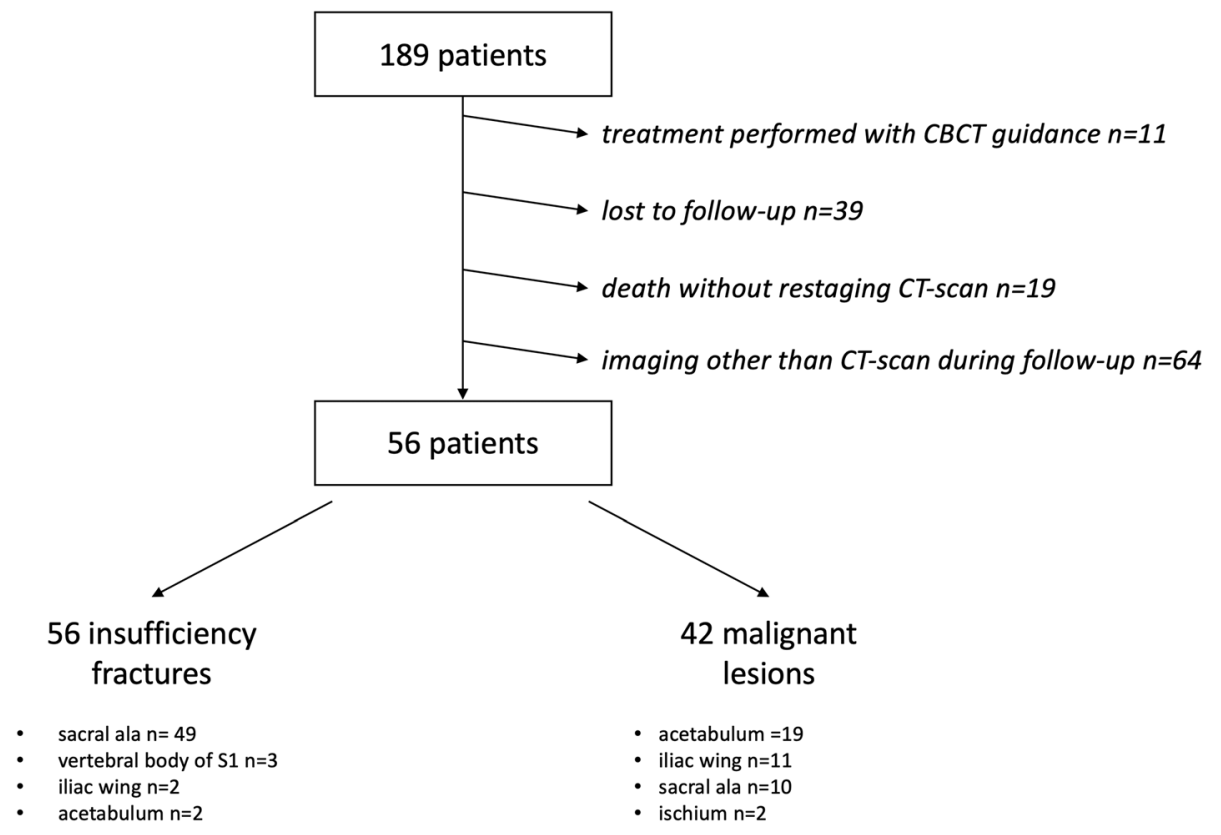

For malignant cases, the osteolysis around the bone cement was classified as follow: stability in 15/42 (35.7\%) cases, partial regression in 13/42 (31\%), complete regression in $9 / 42(21.4 \%)$ and progression in 5/42 (11.9\%).

For insufficiency fractures, radiological signs of consolidation were seen in 53 of the 56 (94.6\%) PMMA injections at last available follow-up, more specifically in 36 of the 39 fractures $(92.3 \%)$ with cortical disruption. There was no fragmentation of PMMA $(0 / 56 \quad 0 \%)$ and no progressive osteolysis around the cement plug during follow-up of insufficiency fractures.
The difference in the rate of fragmentation between the malignant tumor and the benign insufficiency fracture groups did not reach significance $(p=0.08)$.

\section{Discussion}

The present study proposes that fragmentation of the cement plug at midterm follow-up (almost 3 years) is an uncommon finding the following percutaneous injection of PMMA in the bony pelvis. It confirms the data of explanted 


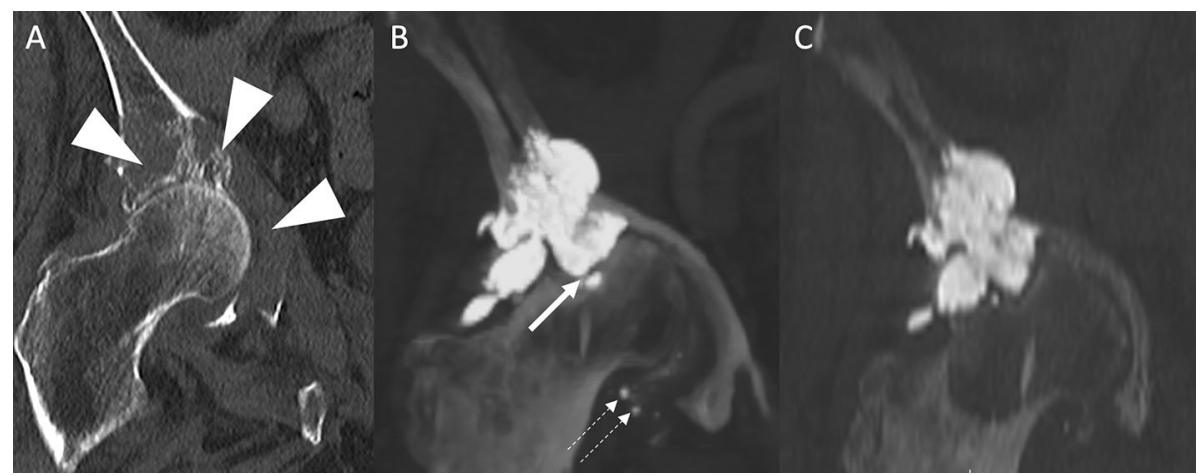

Fig.4 Fragmentation of PMMA. A Coronal CT scan prior to intervention shows an extensive osteolysis of the roof and medial wall of the acetabulum (arrowheads). B 14 months restaging CT scan (coronal view with $15 \mathrm{~mm}$ MIP reconstruction) shows a small fracture at the bottom part of the cement volume (arrow) and PMMA

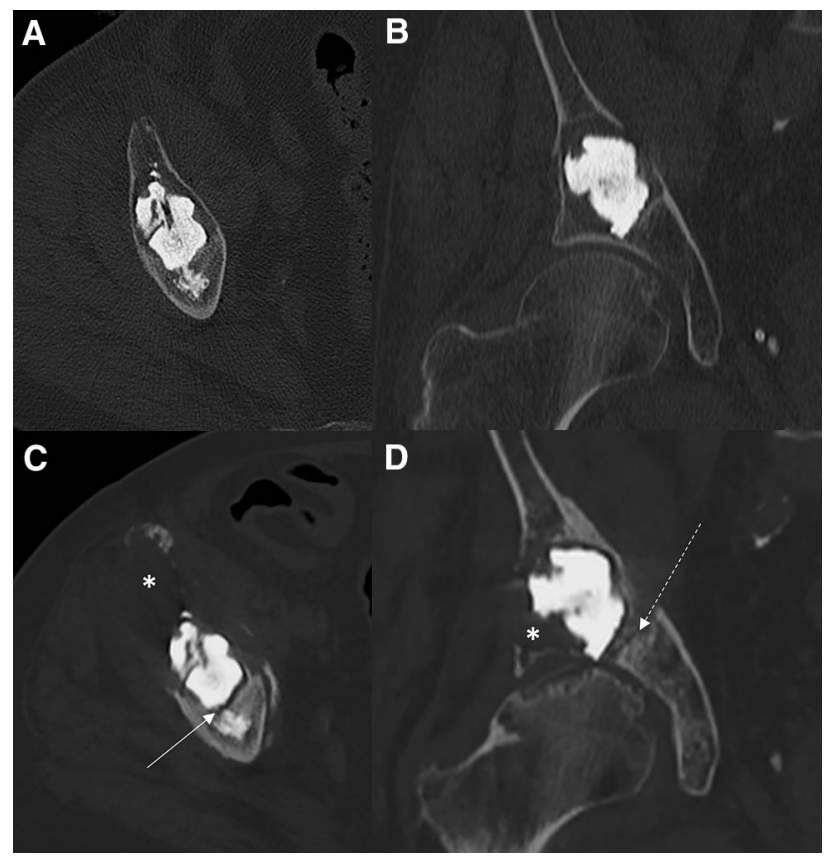

Fig.5 Fragmentation of PMMA. A axial and B coronal CT scan following percutaneous cementoplasty of an acetabular osteolysis. C and D same views from a 42 months follow up CT scan show a fracture inside the cement plug (arrow) compared to baseline. Tumor progression is noted (asterisk) with an apparition of an acetabular fracture (dotted arrow). This case was recorded as fragmentation due to mechanical breakage of PMMA

arthroplasties showing that PMMA undergoes little change once injected in the bone [11]. Based on imaging findings, the two cases of fragmentation likely to have occurred because of failure of the cement to resist complex mechanical stresses, due to extensive osteolysis at the time of treatment for one case and secondary to significant tumor progression with subsequent fracture in the other. The decreased resistance of cement to tension, bending and shear forces compared to compression most certainly inside the joint distant to the initial plug (dotted arrows). Note the deformation of the hip joint with ascension of the femoral head. C Hip deformation was already visible 3 months after cementoplasty. This case was recorded as fragmentation due to mechanical breakage of PMMA

accounts for the occurrence of fragmentation [4]. Such a finding is a known limitation of percutaneous cementoplasty $[13,19,22]$. It may explain why fragmentation occurred only for malignant lesions, which were more likely to deteriorate the resistance of bone and expose PMMA to complex stresses. Hence, PMMA fragmentation in our series seems to be more of a consequence of the local mechanical environment than the cause of further bone collapse/fracture. Similar to radiological features, the lack of clinical improvement at last follow-up is likely due to the worsening of bone deformity and not to the minimal fragmentation of the PMMA plug. The relatively low rate of cement fragmentation in the present report might be explained by the additional use of screw fixation for nondisplaced and displaced pathological fractures as well as for displaced insufficiency sacral fractures since 2013 in our institution. Such combination has been reported to reduce the risk of mechanical failure of stand-alone cementoplasty [22].

While mechanical breakage is related to extrinsic stresses on the cement volume, the pathophysiology of cracking and FBR is more complex and seems to be linked to both extrinsic factors, such as loads and micromotions at the bone cement interface, as well as the working properties of the cement itself such as porosity [23 28]. Porosity is an inevitable process related to the inclusion of air particles inside the PMMA during mixing and injection, leading to micro- $(<1 \mathrm{~mm})$ and macropores $(>1 \mathrm{~mm})$ inside the cement [23]. Although it should be interpreted with great caution, the present data potentially suggest that the porosity of bone cement has little clinical impact for percutaneous procedures at midterm follow-up. Preclinical studies suggest that pores may be the origin of crack initiation that can ultimately lead to fracture and fatigue failure of cement should the cracks propagate [25, 29]. Moreover, pores and cracks are supposed to be a potential 
source for the release of PMMA beads which can subsequently trigger a FBR around the cement [30, 31]. Such reaction usually comes with an extensive osteolysis around the cement and a fragmentation of PMMA [32]. There were no signs suggestive of cracking or FBR in our series. To a certain extent, this further supports that porosity is not clinically relevant, as reported in the orthopedic literature $[28,33]$.

The present study has many limitations. There was no follow-up imaging available for a majority of patients. In our institution, a systematic follow-up CT scan is not organized if the patient remains asymptomatic following cementoplasty. Moreover, many patients were in a palliative situation and died within weeks to months after treatment without restaging imaging. A certain number of patients were also referred from peripheral hospitals and were lost to follow-up following treatment. This might have led to an underestimation in the true rate of PMMA fragmentation. Other causes of underestimation of PMMA fragmentation include the variability of the quality of imaging between baseline and restaging CT scans in some cases. Hence, subtle modifications of the cement volume might have been missed. Finally, there was no histology in the two cases of PMMA fragmentation, in order to rule out formerly infection or FBR. However, the biological analysis and the absence of progressive osteolysis in these two cases were clearly not in favor of infection/FBR. The possibility of spontaneous cement cracking is also unlikely as both cases were associated with major mechanical stresses due to the underlying tumors.

In conclusion, fragmentation of the PMMA plug following percutaneous cementoplasty in the bony pelvis is a rare finding at midterm follow-up. It happened only for cementoplasty performed in malignant lesions and seems to be more of a consequence of the local mechanical stresses than the cause of further bone collapse or fracture.

Funding There was no funding for the present study.

\section{Compliance with Ethical Standards}

Conflict of interest All authors declare that they have no conflict of interest.

Ethical Approval Institutional review board was obtained for the retrospective analysis of the data.

Informed Consent All patient gave informed consent for the procedure.

Consent for Publication All authors/patient gave informed consent for publication of the present data.

\section{References}

1. Zhang HR, Xu MY, Yang XG, et al. Percutaneous vertebral augmentation procedures in the management of spinal metas tases. Cancer Lett. 2020;475:136 42.

2. Garnon J, Meylheuc L, Cazzato RL, et al. Percutaneous extra spinal cementoplasty in patients with cancer: a systematic review of procedural details and clinical outcomes. DiagnInterv Imaging. 2019;100(12):743 52. https://doi.org/10.1016/j.diii.2019.07.005.

3. Clark W, Bird P, Gonski P, et al. Safety and efficacy of verte broplasty for acute painful osteoporotic fractures (VAPOUR): a multicentre, randomised, double blind, placebo controlled trial. Lancet. 2016;388:1408 16.

4. Webb JC, Spencer RF. The role of polymethylmethacrylate bone cement in modern orthopaedic surgery. J Bone Joint Surg Br. 2007;89:851 7.

5. Hesler MC, Buy X, Catena V, et al. Assessment of risk factors for occurrence or worsening of acetabular fracture following percu taneous cementoplasty of acetabulum malignancies. Eur J Radiol. 2019;120:108694.

6. Delpla A, Tselikas L, De Baere T, et al. Preventive vertebroplasty for long term consolidation of vertebral metastases. Cardio vascInterventRadiol. 2019;42:1726 37.

7. Chandra V, Wajswol E, Shukla P, et al. Safety and efficacy of sacroplasty for sacral fractures: a systematic review and meta analysis. J VascIntervRadiol. 2019;30:1845 54.

8. Kurup AN, Schmit GD, Atwell TD, et al. Palliative percutaneous cryoablation and cementoplasty of acetabular metastases: factors affecting pain control and fracture risk. CardiovascInterventRa diol. 2018;41:1735 42 .

9. Turner TM, Urban RM, Singh K, et al. Vertebroplasty comparing injectable calcium phosphate cement compared with poly methylmethacrylate in a unique canine vertebral body large defect model. Spine J. 2008;8:482 7.

10. He Z, Zhai Q, Hu M, et al. Bone cements for percutaneous ver tebroplasty and balloon kyphoplasty: current status and future developments. J OrthopTranslat. 2014;3:1 11.

11. Oonishi $\mathrm{H}$, Akiyama $\mathrm{H}$, Takemoto $\mathrm{M}$, et al. The long term in vivo behavior of polymethyl methacrylate bone cement in total hip arthroplasty. ActaOrthop. 2011;82:553 8.

12. Liao JC, Lai PL, Chen LH, Niu CC. Surgical outcomes of infectious spondylitis after vertebroplasty, and comparisons between pyogenic and tuberculosis. BMC Infect Dis. 2018;18:555.

13. Deschamps F, Farouil G, Hakime A, et al. Cementoplasty of metastases of the proximal femur: is it a safe palliative option? J VascIntervRadiol. 2012;23:1311 6.

14. Klopfleisch R, Jung F. The pathology of the foreign body reaction against biomaterials. J Biomed Mater Res A. 2017;105:927 40.

15. Togawa D, Kovacic JJ, Bauer TW, et al. Radiographic and his tologic findings of vertebral augmentation using polymethyl methacrylate in the primate spine: percutaneous vertebroplasty versus kyphoplasty. Spine. 2006;31:E4 10.

16 Gibon E, Córdova LA, Lu L, et al. The biological response to orthopedic implants for joint replacement. II: Polyethylene, ceramics, PMMA, and the foreign body reaction. J Biomed Mater Res B ApplBiomater. 2017;105:1685 91.

17. Goodman S. Wear particulate and osteolysis. OrthopClin North Am. 2005;36(41 8):vi.

18. Holt G, Murnaghan C, Reilly J, et al. The biology of aseptic osteolysis. ClinOrthopRelat Res. 2007;460:240 52.

19. Yevich S, Tselikas L, Gravel G, et al. Percutaneous cement injection for the palliative treatment of osseous metastases: a technical review. SeminInterventRadiol. 2018;35:268 80. 
20. Huang A, Fang S, Wang L, et al. Vertebral collapse and poly methylmethacrylate breakage after vertebroplasty: a case report. Medicine. 2019;98:e16831.

21. Garnon J, Meylheuc L, De Marini P, et al. Subjective analysis of the filling of an acetabularosteolytic lesion following percuta neous cementoplasty: is it reliable? CardiovascInterventRadiol. 2020;43(3):445 52. https://doi.org/10.1007/s0027001902397 1.

22. Lea WB, Neilson JC, King DM, et al. Minimally invasive sta bilization using screws and cement for pelvic metastases: tech nical considerations for the pelvic "screw and glue" technique. SeminInterventRadiol. 2019;36:229 40.

23 Lewis G. Properties of acrylic bone cement: state of the art review. J Biomed Mater Res. 1997;38:155 82.

24. Janssen D, Aquarius R, Stolk J, et al. The contradictory effects of pores on fatigue cracking of bone cement. J Biomed Mater Res B ApplBiomater. 2005;74:747 53.

25. James SP, Jasty M, Davies J, et al. A fractographic investigation of PMMA bone cement focusing on the relationship between porosity reduction and increased fatigue life. J Biomed Mater Res. 1992;26:651 62.

26. Willert HG, Bertram H, Buchhorn GH. Osteolysis in alloarthro plasty of the hip. The role of bone cement fragmentation. Clin Orthop Relat Res. 1990; pp 10821.

27. Zhang H, Blunt L, Jiang $X$, et al. The significance of the micropores at the stem cement interface in total hip replacement. J BiomaterSciPolym Ed. 2011;22:845 56.
28 Ling RS, Lee AJ. Porosity reduction in acrylic cement is clinically irrelevant. ClinOrthopRelat Res. 1998;355:249 53.

29. Fritsch E, Rupp S, Kaltenkirchen N. Does vacuum mixing improve the fatigue properties of high viscosity poly(methyl methacrylate) (PMMA) bone cement? Comparison between two different evacuation methods. Arch Orthop Trauma Surg. 1996;115:131 5

30. Ries MD, Young E, Al Marashi L, et al. In vivo behavior of acrylic bone cement in total hip arthroplasty. Biomaterials. 2006;27:256 61 .

31. Shardlow DL, Stone MH, Ingham E, et al. Cement particles containing radio opacifiers stimulate pro osteolytic cytokine production from a human monocytic cell line. J Bone Joint Surg Br. 2003;85:900 5.

32. Slullitel PAI, Brandariz R, Onativia JI, et al. Aggressive granu lomatosis of the hip: a forgotten mode of aseptic failure. IntOr thop. 2019;43:1321 8.

33. Janssen D, Stolk J, Verdonschot N. Why would cement porosity reduction be clinically irrelevant, while experimental data show the contrary. J Orthop Res. 2005;23:691 7. 